\title{
LACK OF ACCESS TO GENETIC TESTING AMONG PATIENTS AT RISK OF HEREDITARY BREAST CANCER IN THE BRAZILIAN PUBLIC HEALTH SYSTEM
}

Tatiana Strava Corrêa', Renata Lazari Sandoval', Luiza Nardin Weis³, Ana Carolina Rathsam Leitee,2, Romualdo Barroso de Sousa',3

${ }^{1}$ Hospital Sírio-Libanês, Centro de Oncologia de Brasília - Brasília (DF), Brazil.

${ }^{2}$ Hospital Materno Infantil de Brasília - Brasília (DF), Brazil.

3Instituto Hospital de Base do Distrito Federal - Brasília (DF), Brazil.

Objectives: In Brazil, patients treated in the public health (PH) system have no access to genetic testing. This study aims to explore the clinical profile of patients at risk of hereditary breast cancer (BC) in a tertiary hospital at the Federal District. Methodology: Patients with a previous diagnosis of BC, in treatment or follow-up at our PH service, were evaluated for the risk of hereditary BC from January to March 2021. Patients who fulfilled criteria for genetic testing, according to the National Comprehensive Cancer Network (NCCN) criteria (version 1.2020), were defined as at risk of hereditary BC. The clinical data were collected after signed informed consent. Results: A total of 70 patients were evaluated, and 51 patients who fulfilled the NCCN criteria for genetic testing were included in this analysis. The median age at BC diagnosis was 42 years. Invasive ductal carcinoma represented $96 \%$ of the cases. The positive hormonal receptor was present in $70.6 \%$ of the tumors (36/51) and HER-2 enriched tumors in $19.6 \%$. Ki67 14\% was found in 82.3\% (42/51) of cases. Tumors were grade $2(80 \%)$ and grade $3(13.7 \%)$. Most patients were diagnosed at locally advanced stages: $62.7 \%$ stage IIBIIIC, $22 \%$ stage IV, and 13.7\% IA-IIA. The most frequent NCCN criteria for the hereditary BC investigation were BC diagnosis $\leq 45$ years (66\%), family history of breast/prostate/pancreatic cancer $(60 \%)$, and triple-negative BC <60 years $(25 \%)$. Only four patients performed genetic testing at their own costs. Two of them had positive test results (BRCA2 and TP53 pathogenic variants). Conclusion: This analysis showed that a high rate of patients with $\mathrm{BC}$, who have the indication of genetic testing, have locally advanced or metastatic disease at the time of BC diagnosis. This is likely to lead to worst disease outcomes. Considering that approximately $10 \%$ of BC cases are associated with cancer predisposition syndromes, we expect that at least 5 of 51 evaluated patients would have positive genetic tests, which would allow the risk-reduction strategies for secondary cancers and the identification of other family members at risk.

Keywords: Hereditary Breast Cancer; Public Health; Genetic Testing. 